\title{
Contextual Sentiment Based Recommender System to Provide Recommendation in the Electronic Products Domain
}

\author{
N. A. Osman, S. A. M. Noah, and M. Darwich
}

\begin{abstract}
The rush to purchase the latest products sometimes prevents people from thinking things through completely. Consequently, recommender services are increasingly emerging. By looking at industry trends, interviewing dozens of leading industry stakeholders, and using publicly available information, it is important to filter out the most relevant information for consumer electronics before purchasing their items. This paper presents an electronic product recommender system based on contextual information from sentiment analysis. The recommendation algorithms mostly rely on users' rating to make prediction of items. Such ratings are usually insufficient and very limited. We present a contextual information sentiment based model for recommender system by making use of user comments and preferences to provide a recommendation. The purpose of this approach is to avoid term ambiguity which is so called domain sensitivity problem in recommendation. The proposed contextual information sentiment-based model illustrates better performance by using results of RMSE and MAE measurements as compared to the conventional collaborative filtering approach in electronic product recommendation.
\end{abstract}

Index Terms-Collaborative filtering, recommender systems, sentiment analysis, electronic product, domain sensitivity.

\section{INTRODUCTION}

Products recommendations have become increasingly popular in e-commerce services such as in Amazon Instant Video service and Netflix [1]. The vast growth of electronic products e-commerce has made online commerce face new challenging situations. The companies in the electronic e-commerce have found it harder to survive due increasing competitions. On the other hand, customers have been overloaded with information of electronic products where they are no longer able to effectively choose them. As a result concern with new marketing strategies such as one-to-one marketing and customer relationship management (CRM) are growing [2]. One of the promising technologies to overcome product overload and a new marketing strategy solution is the product recommendation method in recommender systems.

The goal of a recommender system (RS) is to generate meaningful recommendations to a collection of users for items or products that might interest them [3]. The use of electronic product recommendation systems is ubiquitous among large e-commerce companies today. RSs have proven

Manuscript received November 18, 2018; revised March 25, 2019. This work was supported in part by the Malaysia Ministry of Education Grant FRGS/1/2014/ICT02/UKM/01/1.

The authors are with the Universiti Kebangsaan Malaysia, Bangi, Selangor, Malaysia (e-mail: n.aidaosman@gmail.com, shahrul@ukm.edu.my, modarwish@hotmail.com). to be one of the most powerful and popular tools in product recommendation as illustrated by Amazon.com and eBay, which employ an RS to personalize the online store for each individual customer [4].

The collaborative filtering technique is one of the most successful techniques used by recommender system which filters information by exploiting the recommendation of other similar users. This technique recommend items to a user based on similarities between the past behavior of the user and that of likeminded people [5]. Collaborative filtering is also the most successful recommendation technology but its application to e-commerce has exposed well-known limitations such as sparsity [6] and scalability. This is due to the fact that recommendation algorithms mostly rely on users' ratings to make prediction of items. Such ratings are usually insufficient and very limited

Sentiment analysis algorithms offer an analysis of the user's preferences in which the comments may not be associated with an explicit rating. Hence, embedding sentiment in recommender systems may enhance the recommendation quality of recommender systems. It will have an impact on the popularity of the recommendation item. Thus, sentiment analysis is used to determine the words or sentences that have sentiment value [7]. However, the conventional sentiment analysis method in recommender system suffers from a term ambiguity problem called domain sensitivity. Domain sensitivity problem in recommender systems can be vanquished by elevating contextual information in conventional sentiment analysis model. As a result, this information can be used to improve the performance of current recommender systems. The current sentiment intensity enhanced with the contextual information will reduce the ambiguity of opinion words in different domains and will thus improve the overall recommendation quality.

Hence, this paper aims to provide the contribution of contextual information sentiment analysis to electronic product recommender systems. This paper provides a solution to minimize the data sparsity and improve the RMSE and MAE value in electronic product recommender systems by integrating unrated reviews and ratings.

\section{RELATED WORKS}

\section{A. Recommender Systems}

Recommender Systems (RSs) are software tools and techniques that provide suggestions for items that are most likely of interest to a particular user [8]-[10]. The suggestions are related to various decision-making processes, such as 
what items to buy, what music to listen to, or what online news to read [11]. RSs are mainly directed toward individuals who are not competent to evaluate the relevant large number of optional items that a website may suggest [10]. Recommender systems differ in the way they analyze these data sources to develop notions of similarity between users and items which can be used to identify well-matched pairs. Recommender systems are usually classified into the three categories according to the approach of recommendations [12] which are content-based recommendation, collaborative recommendation and hybrid approaches.

Collaborative Filtering (CF) based recommender systems have been proven to be a promising solution to the problem of information overload. Such systems provide personalized recommendations to users based on their previously expressed preferences and that of other similar users. It is the most popular technique and algorithm in RSs. However, besides the popularity of $\mathrm{CF}$, to a certain extent, it could not recognize the preferences of users in cold-start scenarios, where insufficient preferences from users or items will lead to degraded recommendation quality. Ghabayen and Noah [13] has proposed a solution to overcome the cold-start recommendation problem by exploiting social tags in their works. CF analyze historical interactions alone. A few examples selected from different domains are introduced in this section. Amazon.com [4] is one of the most successful and well-known online retailers. It makes use of purchase histories of customers to produce recommendations using the item-based technique. GroupLens [9], [14] is a pioneering and well-known project in automated CF. It had used k-Nearest Neighbour to do the recommendation. Filtering agents were later integrated into the system to improve prediction quality [15].

Content-based filtering systems are based on profile attributes. Content-based filtering is prevalent in information retrieval, where the text and multimedia content of documents is used to select documents relevant to a user's query. In the context of recommender systems, this refers to content-based recommenders which provide recommendations by comparing representations of content describing an item to representations of content that interests a user [3], [16].

Hybrid techniques attempt to combine both of these designs. In CF recommender systems, ratings which indicates how a particular user liked particular items have been the most popular representation to date [17]. However, ratings have limitations particularly when the dimension of user-item matrix increases. This may result in the data sparsity problem. As a result, few research works have been done to overcome the above-mentioned problem [18].

In recent years, $\mathrm{CF}$ has gained research attention in the electronic product domain for several reasons. This is particular for valuable reasons such as material choice and design, evaluating and selecting software compatibility, and many others. Besides, one characteristic of the purchase of electronic products is that at the moment of decision making,

only information about the product, not the product itself, is available to users [19]-[21].

JunBo Xia [22] discussed an e-commerce product recommendation method based on collaborative filtering technology. In his paper, he exploited collaborative filtering algorithm to recommend products for users and proved that the proposed method can recommend more relevant products for users with high accuracy.

\section{B. Sentiment Analysis}

Sentiment Analysis (SA) involves the detection and extraction of sentiment, attitude and opinion from text. SA is widely applied in many domains, including commercial products and services, politics, stock market prediction and customer relationship management [23]. SA generally will analyze the structure of textual review then will interpret it in the form of positive or negative sentiment. However, within the context of recommender systems, sentiment must be correlated with ratings. In other words, it is not sufficient to classify specific statements as positive or negative, but they must be correlated with such statements in the form of numerical ratings. One of the most important tasks in sentiment analysis is to identify which words express a sentiment as in [24]. SentiWordNet dictionary is a popular linguistic resource in sentiment analysis which provides an answer to the "how and which words people use to express preferences?" as mentioned in [21]. Similar sentiment dictionaries have also been automatically created for other languages [25], [26]. SA also been used to deal with crossdomain problem. Al-Molsmi et al. [27] has tackled this issue to improve cross-domain sentiment classification by creating a resource in the form of an overview of the techniques, methods, and approaches that have been used in order to assist researchers in developing new and more accurate techniques in the future. There are few research works that are related to sentiment analysis on product reviews that were studied in this paper. Nisha and Kirubakaran [24] did their survey on sentiment analysis of product reviews. Their paper provides an overall survey about sentiment analysis or opinion mining related to product reviews. Hu et al., [28] performed mining and summarization process to all the customer reviews of a product.

\section{Sentiment Based Approach in Product Recommendation}

The aim of this paper is to integrate sentiment analysis in product recommender systems especially to those items with no associated rating which basically will lead to the problem of data-sparsity and domain sensitivity. Based on the existing literature on product recommendation system and sentiment-based recommender systems, most approaches depend on conventional sentiment-analysis approaches. The conventional approaches consist of only a general lexicon that lacks of semantic and contextual information.

Some related applications in the product domain were also studied. They are mainly based on knowledge based technologies. CF is only used to rank the recommended products according to user preferences. Chun and Hong [5] implemented knowledge-based recommender system for electronic commerce. They designed an implementation of product recommender system on an Internet shopping mall. The knowledge base of product domain and inference engine was implemented by JESS and Java servlet. Their system gathered the user's requirements on a particular product by questioning the user and consulting its knowledge base to find the items that best meet the user's requirement.

There are several other research works that incorporated 
sentiment analysis in recommender system. A research by [29] discussed about recommendation system using sentiment analysis considering the polarity of adverbs. Shapira et al. [11] also discussed on the introduction and challenges in recommender system which was directed to the solution of applying sentiment analysis in recommender systems. One of the interesting research works that focuses on context sensitivity in recommender system was done by Alhamid et al. [20]. They discussed towards context sensitive collaborative media in recommender system by incorporating social tags and rating information. Another interesting research that applied sentiment analysis technique in recommender system is done by [7]. His paper presented a detailed explanation on recommender system for the TV on the web by integrating unrated reviews and movie ratings.

Those research works basically explained the methods and solutions applied to solve the ultimate problems in recommender systems which are cold-start and data sparsity. Sentiment analysis technique seems to be an alternative solution to tackle out the issues by making use of the textual reviews. Sentiment analysis technique can be enhanced to overcome the domain sensitivity problem by leveraging contextual sentiment based model for recommender systems.

\section{Proposed ConteXtual Sentiment BASED RECOMMENDATION MODEL}

The proposed contextual sentiment based recommendation model aims mainly to enhance the effectiveness of collaborative recommender systems by integrating textual reviews into the user-item ratings matrix. Hypothetically, the integration of textual review with rating data can reduce the main problem of recommender systems, which are data sparsity and consequently improved the quality of recommendation. Besides, the proposed model will surmount the issue of domain sensitivity in recommender systems. The conventional sentiment based model for product recommender systems will be enhanced by incorporating contextual information component.

\section{A. Merging Textual Reviews with Rating Data}

One of the solutions to deal with the data sparsity problem in recommender system is by merging textual reviews with rating data using sentiment analysis technique. Research in sentiment analysis has shown that such information can produce better sentiment accuracy and help in improving the data sparsity problem. Peleja et al [7] proposed a technique of sentiment analysis in integrating unrated reviews and movie ratings for TV recommender systems. They have proved that the proposed recommendation framework can successfully integrate unrated reviews with ratings to improve ratings-only recommendations. However, the effectiveness of how such domain sensitivity information can improve the quality of recommendation is yet to be explored. Our work exploited the conventional sentiment based model for recommendation system by adding domain sensitivity information component.

A major problem restraining the usefulness of collaborative filtering is the data sparsity problem, which refers to a situation in which feedback data is sparse and insufficient to identify similarities in user interests. Fig. 1 shows the issue of data sparsity in recommender systems and how sentiment analysis technique helps to solve the raised problem.

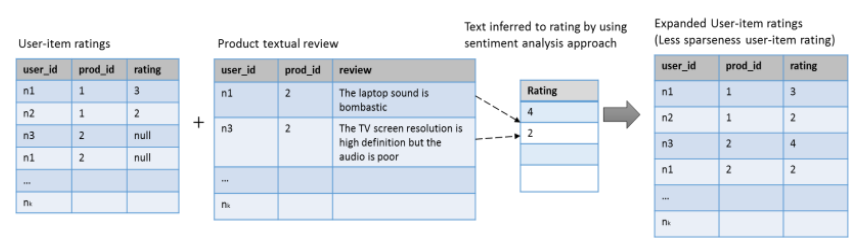

Fig. 1. Merging ratings and textual reviews can improve data sparsity problem.

Fig. 1 shows that merging ratings and textual reviews can reduce the value of data sparsity in recommender systems. The quality of recommender systems can be improved compared to ratings only recommender systems.

Our work further explored on the domain sensitivity issue in recommender systems. Based on the existing works on sentiment-based recommender systems, most approaches depend on conventional recommender techniques that lack of semantic value which will lead to domain sensitivity problem. The conventional sentiment analysis based model in recommender system uses only general lexicon in sentiment model. Thus, the ambiguity opinion words might have occurred in different domains such as products and movies domain.

The following is an example for a same opinion word such as the word "sucks". It will reflect a positive sentiment for a sentence in the product domain e.g "this vacuum sucks very well", while for a sentence in the movie domain e.g "I hate this story. The actor's role is sucks" reflect a negative sentiment. Thus, embedding such enhanced-sentiment approaches were investigated and implemented in sentiment-based recommender systems. Fig. 2 shows an overall flow process of contextual information based sentiment analysis in product recommender system.

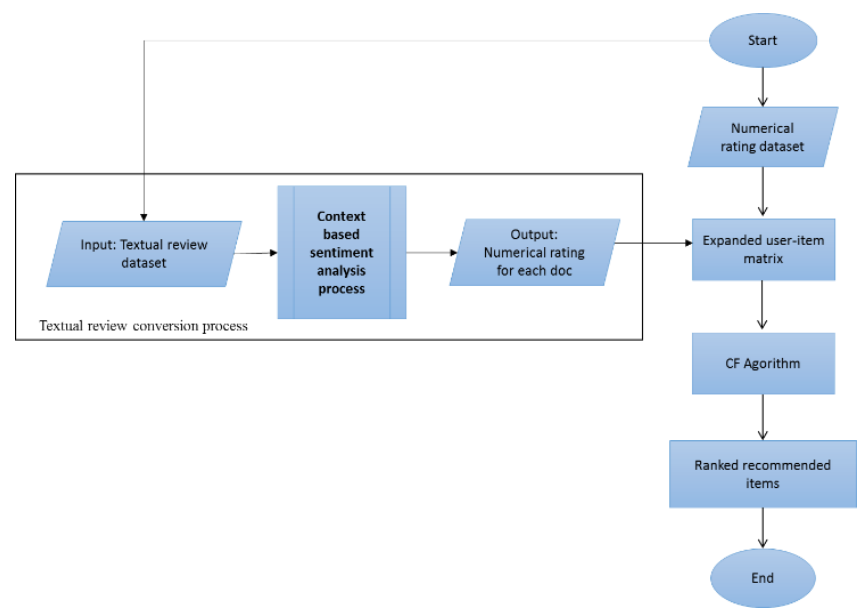

Fig. 2. The overall flow process of context based sentiment analysis of collaborative recommendation.

Fig. 2 depicts the overall flow process of context based sentiment analysis recommender system. The process started with a textual review conversion process. The conversion process started with the input of textual reviews from the product dataset. Sentiment analysis technique will be applied during the conversion process of unrated textual review to rating data. The common text processing was applied during the process. The processes include text splitting, part of 
speech (POS) tagging, and then enriching the POS-tagged text with our own tags using dictionaries. Then, some basic extraction rules over the tagged text were applied. Each review will be converted into a numerical rating by using sentiment analysis technique. The numerical rating output were then combined with the existing numerical rating dataset in the same format. The combination from both dataset will produce the expanded user-item matrix. From the expanded user-item matrix that contains both ratings from reviews and rated data, it is fed into the $\mathrm{CF}$ algorithm to calculate user's weight and similarities. The calculation for each recommendation is based on the collaborative recommendation equation as follows:

$$
\hat{\boldsymbol{r}}_{u i}=\boldsymbol{b}_{u i}+\sum_{j \in D_{c}^{k}(u: i)} \boldsymbol{d}_{i j}\left(\boldsymbol{r}_{u j}^{\prime}-\boldsymbol{b}_{u j}\right)
$$

where $\hat{\boldsymbol{r}}_{\boldsymbol{u} i}$ estimates the rating of a user $u$ for an unseen item $i$. It relies on the bias estimate $b_{u i}$ of the user $u$ for various items $j$ and on a score computed using the $k$ most similar items to $i$ that the user $u$ has already rated. The neighbourhood $D_{c}^{k}(u ; i)$ is the neighbourhood of the $\mathrm{k}$ most similar items that the user has already rated or commented and $r_{u j}^{\prime}$ is the result of the mapping function that accounts for both explicit ratings and those inferred from comments.

The implication of applying a domain-sensitive sentiment lexicon for the model is emphasized by the following real-world example. The sentence "this laptop is very light. I carry it to school every day as if my bag is empty" would be labelled as neutral. There are no explicit sentiment words in the sentence. However, using a domain sensitive lexicon that has been adapted to the electronic products domain, the sentence would be correctly flagged with a positive polarity, as the writer is talking about how the laptop has a light weight This domain adaptation step would fine-tune the sentiment lexicon to tag terms with a polarity based on the specific domain itself, and in turn improve the overall accuracy of the sentiment analysis model.

$\mathrm{n}$ context based sentiment analysis process, the first process begins with the extraction of all potential opinion words (OW) from the corpus. The focus is only on adjectives, verb phrases and noun phrases. The Stanford SNAP Electronic Product Reviews corpus (https://snap.stanford.edu/data/web-Amazon.html) which contains about 1 million reviews was used for this task, as shown in Fig. 3. The sub processes of the context based sentiment analysis process were detailed out in Fig. 3.

In the second process, from the generated OW list, a set of predefined positive and negative seed words were measured. Example of positive seed words are great, happy, nice, and beautiful, whereas example of negative seed terms are poor, worst, terrible, and ugly. For each of the seed word would generate a sentiment score in the range of $[+1,-1]$. The closer the $\mathrm{OW}$ is to one end of the spectrum, the higher its sentiment strength.

In the third process, the linguistic rules like "OR" and "AND" will be applied. The "AND" rule will join two adjectives of the OWs with similar sentiment and the "BUT" rule will join two adjectives with different sentiment. For all of the OW AND POS_SEED_WORD, OW BUT POS_SEED_WORD, OW AND NEG_SEED_WORD, and OW BUT NEG_SEED_WORD from the corpus were taken, and use these occurrences of the OWs and the SEED WORDS found within these linguistic constraints and from them a sentiment score are generated. These rules only work on adjectives, so scores for adjective OWs using this technique were only generated.

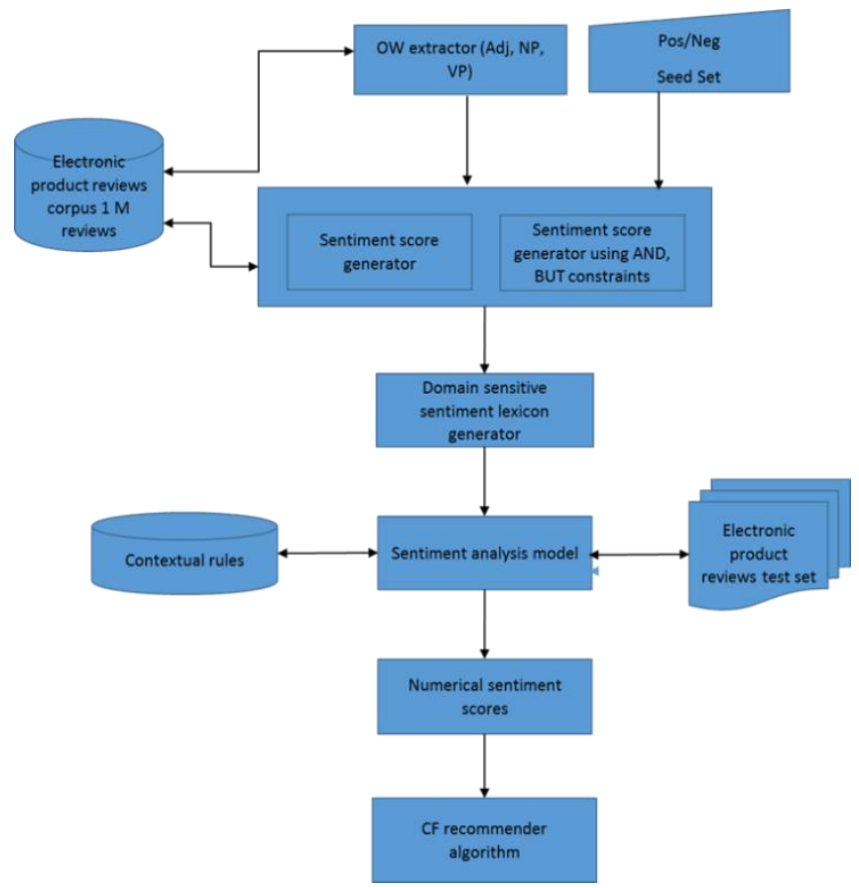

Fig. 3. The enhanced context based sentiment analysis process consisting of the following sub processes.

Next, the scores of adjectives from the second and third process were combined, and the scores of noun and verb phrases generated in the second process will be used. The generated sentiment lexicon is sensitive to the domain of the corpus. The sentiment lexicon generator combined the score from the two sentiment scores above it. It just took the average of each score for each word. The contextual rules would be useful to improve accuracy which are negations such as not and no, intensifiers such as very and extremely and diminishers such as slightly, hardly, minimally. So if an electronic product reviews corpus was used, the lexicon generated would best perform on an electronic product reviews test set.

Algorithm 1 described the process of converting textual review into sentiment rating. The resulting algorithm makes use of four contextual rules lists: NegationList, PosList, NegList and IntensifyList. PosList and NegList respectively contain all the positive and negative lexicons. NegationList contains the inverse lexicons such as not, do not and cannot, whereas IntensifyList includes increment terms such as very and extremely and decrement terms such as little and decrease. These general opinion lexicons were taken from Bing Liu lexicons [28].

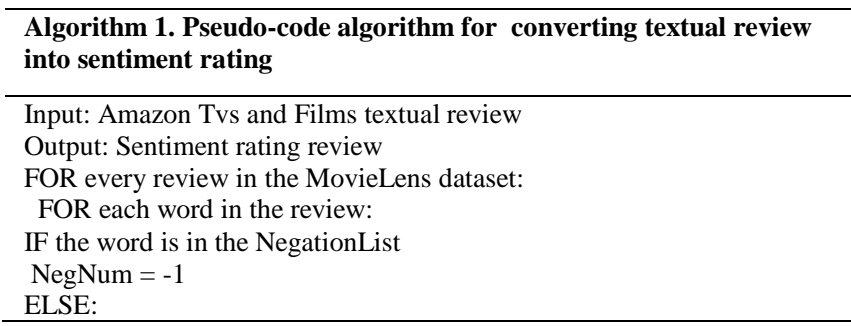




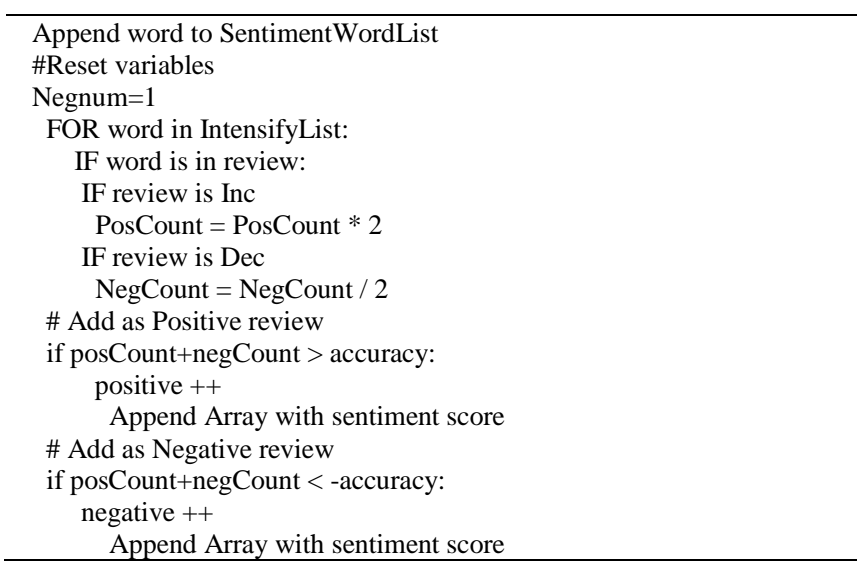

The measurement of the proposed model will be measured by accuracy value. The accuracy value of the domain sensitivity generated lexicons will be compared to the general lexicon such as Bing Liu Lexicon with the generated domain sensitivity lexicon. The accuracy value for both models will be measured. The contextual information are considered to further improve accuracy value.

Finally, the numerical scores for the electronic product dataset documents can be used as the input for $\mathrm{CF}$ recommender system and then it would rank and recommend product to the user based on their sentiment score and rating toward each product.

\section{B. Integration of Sentiment Rating with Actual Rating}

To establish a contextual information sentiment-based recommendation model, sentiment ratings obtained from the previous process as explained in Section III.A were then fed into the recommender system framework as shown in Fig. 2. Some of the products in the dataset derived might have duplication between the actual rating and sentiment rating. Thus, (2) was formulized to remove any duplication for the same product between actual rating and sentiment rating. The standard weightage for each of the duplicated product is calculated.

The value of sentiment rating and numerical rating are then combined to get the overall rating as illustrated in (2):

$$
P=\lambda \text { ActRating }+(1-\lambda) \text { SentRating }
$$

where ActRating is the actual rating provided by users and SentRating is the sentiment rating derived from users' reviews. $\lambda$ is a damping factor that decides the contribution of the individual ratings. During experiments we explore three values of $\delta$ weightage which are $0.3,0.5$ and 0.7 .

\section{RESUltS AND EVALUATION}

The experiment was performed on a standard available dataset derived from Amazon Dataset (http://jmcauley.ucsd.edu/data/amazon/links.html). The dataset contains about 2000 reviews and 5000 rating from the electronic products. The baseline data set consists of 5,000 ratings (scaled between 1 and 5) from 8,773 users on 468 electronic products. A total of 2,000 textual reviews from 468 products were added into the baseline dataset. The data then were cleansed and the duplication ratings for the same products were calculated by using (2). The standard measurement of root-mean-square error (RMSE) and mean absolute error (MAE) were calculated to compare the effectiveness of each model.

The objective of the experiment is to measure the effectiveness performance of recommender systems by using both conventional sentiment analysis based model and contextual information based model in recommender system. The value of RMSE and MAE between both models were compared. For conventional sentiment analysis based recommendation model, Bing Liu Lexicons was used as a baseline. The contextual information sentiment based recommendation model was then expanded by the generated domain sensitivity lexicon.

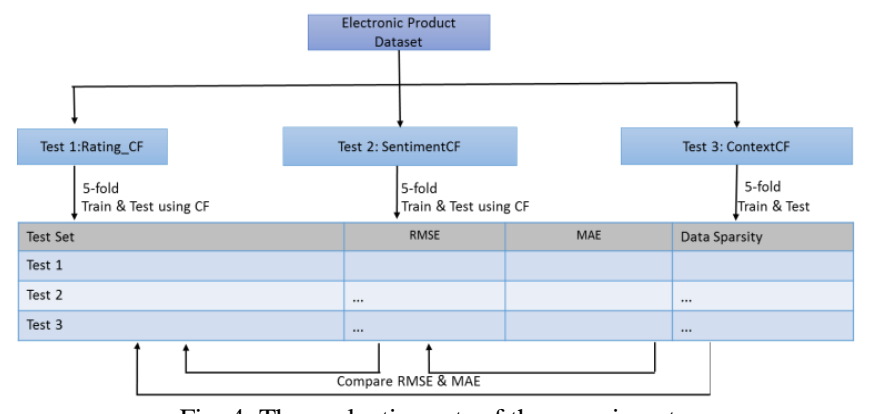

Fig. 4. The evaluation sets of the experiments.

As shown in Fig. 4, there are three different sets of experiments performed by classifying them into three sets of tests; Test 1 , Test 2 and Test 3 . Test 1 is the the conventional ratings-based $\mathrm{CF}$ model, Test 2 is the enhanced $\mathrm{CF}$ model with sentiment ratings and Test 3 is the $\mathrm{CF}$ model with contextual sentiment ratings. We called these models as ratings-based $\mathrm{CF}$ (ratingCF), sentiment $\mathrm{CF}$ (sentimentCF) and contextual sentiment $\mathrm{CF}$ (contextCF) respectively. The ratingCF and sentimentCF will be the baseline for the experiment. In this experiment, we used Bing Liu lexicons as a baseline. The lexicons later were expanded in the contextCF with the specific domain lexicon generated. Each of the sets were tested to acquire the value of RMSE, MAE and Sparsity.

RMSE and MAE are used to measure the value of error rate in recommendation. MAE [30] and RMSE are usually used as statistical accuracy metrics. MAE is the most popular and commonly used. It is a measure of deviation of recommendation from user's specific value. It is computed as follows [31]:

$$
M A E=\frac{1}{N} \sum_{u, i}\left|P_{u, i}-r_{u, i}\right|
$$

where $P_{u, i}$ is the predicted rating for user $u$ on item $i, r_{u, i}$ is the actual rating and $N$ is the total number of ratings on the item set. The lower the MAE, the more accurately the recommendation engine predicts user ratings. RMSE puts more emphasis on larger absolute error and the lower the RMSE is, the better the recommendation accuracy. Also, the RMSE is given by Cotter et al. [32] as (4):

$$
R M S E=\frac{1}{N} \sum_{u, i}\left(P_{u, i}-r_{u, i}\right)^{2}
$$

While data sparsity can be measured by using (5):

$$
\text { Sparsity }=100 \% \text { - Density }
$$


where;

$$
\text { Density }=\frac{\text { Number of Ratings in the Dataset }}{(\text { Number of movies }) *(\text { Number of Users })} \times 100 \%
$$

The sum of the sparsity and density levels should equal to $100 \%$. Higher sparsity levels means more data are not filled with the rating value. The higher density levels means the lesser value of data sparsity should be. The data sparsity levels in the electronic product dataset used is $99.80 \%$ which is quite high. High sparsity levels pose a great challenge to the quality of predictions, as well as to the number of predictions that a recommender system can actually compute [33]. The quality of collaborative filtering recommendations is highly dependent on the sparsity of available data [34]. In the common case, as the sparsity increases, CF algorithms may start to deteriorate as they are unable to form reliable neighbourhoods.

To measure the best performance of RMSE and MAE for the proposed model, different factor $\lambda$ of the ratings for sentimentCF and contextCF were assessed. Based on (2), the initial experiment's results for three different values of $\lambda$ was recorded in Table I.

TABLE I: RESULTS USING DIFFERENT $\lambda$ FACTOR

\begin{tabular}{lrrrr}
\hline \hline & \multicolumn{2}{c}{ SentimentCF } & \multicolumn{2}{c}{ contextCF } \\
\hline & RMSE & MAE & RMSE & MAE \\
\hline 0.7 & 4.0950 & 3.9049 & $\mathbf{4 . 0 1 9 7}$ & $\mathbf{3 . 7 7 9 8}$ \\
0.5 & 4.1182 & 3.9338 & 4.0596 & 3.8202 \\
0.3 & 4.1460 & 3.9642 & 4.0361 & 3.7861 \\
\hline \hline
\end{tabular}

The results showed that the best performance was achieved for $\lambda=0.7$ which indicates that the actRating contribute to the best result of both sentimentCF and contextCF. Hence, $\lambda=$ 0.7 was used in the remaining experiments.

A further experiment was performed to test if the proposed model can really improve the sparsity level. The sparsity level also decreased from $99.80 \%$ to $99.70 \%$ as plotted in Fig. 5 .

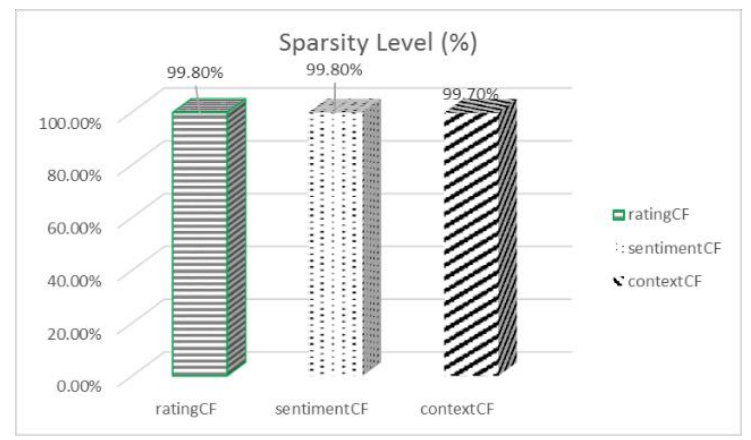

Fig. 5. Sparsity levels for the three CF models.

Fig. 5 shows that the sparsity level decreased using our proposed model compared to the ratingCF and sentimentCF models.

\section{CONCLUSION}

As opposed to other domains, electronic product recommendation system should be built up according to the special features of a certain sort of product, and forming professional recommendation systems for different features of electronic products. The limitations of the ambiguous word in the electronic product review features can be resolved by the help of the contextual information. The contextual information sentiment based model for product recommender systems has tackled this issue. For example, consider the word "unpredictable". A review like "unpredictable sound" gives positive sentiment yet another review like "unpredictable steering" gives the opposite sentiment.

The proposed model which optimized the conventional sentiment based model has proven to gain the best RMSE and MAE value of recommendation model and minimized the level of data sparsity. Hypothetically, the proposed model of contextual information sentiment-based recommendation algorithm enhancing the performance of electronic product recommender systems has been achieved. Future works include implementation and evaluation for the other domains such as medicine.

\section{ACKNOWLEDGMENT}

This research is partially supported by the Universiti Kebangsaan Malaysia under the Grand Challege Fund DCP-2017-002/3 and the Malaysia Ministry of Education Grant FRGS/1/2014/ICT02/UKM/01/1 awarded to the Centre for Artificial Intelligence Technology (CAIT).

\section{REFERENCES}

[1] R. Bell, Y. Koren and C. Volinsky, "Matrix factorization techniques for recommender systems," Computer, vol. 42, no. 8, pp. 30-37, 2009.

[2] D. H. Ahn, J. K. K. Kim, and Y. H. Cho. "A comparative evaluation of hybrid product recommendation procedures for web retailers," presented at 9th Asia-Pacific Decision Science Institute Conference, Korea, 2004.

[3] P. Melville and V. Sindhwani, "Recommender systems," Encyclopedia of Machine Learning, Springer, Boston, MA, pp. 829-838, 2011.

[4] G. Linden, B. Smith, and J. York, "Amazon. com recommendations: Item-to-item collaborative filtering," IEEE Internet Computing, vol. 7, no. 1, pp.76-80, February 2003.

[5] I. G. Chun and I. S. Hong, "The implementation of knowledge-based recommender system for electronic commerce using Java expert system library," in Proc. IEEE International Symposium on Industrial Electronics, 2001, vol. 3, pp. 1766-1770.

[6] X. Su and T. M. Khoshgoftaar, "A survey of collaborative filtering techniques," Advances in Artificial Intelligence, p. 19, 2009.

[7] F. Peleja, P. Dias, F. Martins, and J. Magalhães, "A recommender system for the TV on the web: integrating unrated reviews and movie ratings," Multimedia Systems, vol. 19, no. 6, pp. 543-558, 2013.

[8] R. Burke, "Hybrid web recommender systems," The Adaptive Web, Springer, Berlin, Heidelberg, pp. 377-408, 2007.

[9] P. Resnick, N. Iacovou, M. Suchak, P. Bergstrom, and J. Riedl, "GroupLens: An open architecture for collaborative filtering of netnews," in Proc. the 1994 ACM Conference on Computer Supported Cooperative Work, ACM, 1994, pp. 175-186.

[10] P. Resnickand and H. R. Varian, "Recommender systems," Communications of the ACM, vol. 40, no. 3, pp. 56-58, 1997.

[11] F. Ricci, L. Rokach, and B. Shapira, "Recommender systems: introduction and challenges," Recommender Systems Handbook, Springer, Boston, MA, pp. 1-34, 2015.

[12] D. Jannach, M. Zanker, A. Flfering, and G. Friedfrich, Recommender Systems: An Introduction, New York: Cambridge University Press, 2011.

[13] A. S. Ghabayen and S. A. Noah, "Exploiting social tags to overcome cold start recommendation problem," Journal of Computer Science, vol. 10, no. 7, pp. 1166-1173, 2014.

[14] J. A. Konstan, B. N. Miller, B. N. Maltz, J. L. Herlocker, L. R. Gordon, and J. Riedl, "GroupLens: Applying collaborative filtering to Usenet news," Magazine Communications of the ACM, vol. 40, no. 3, pp. 77-87, 1997.

[15] B. M. Sarwar, J. A. Konstan, A. Borchers, J. Herlocker, B. Miller, and J. Riedl, "Using filtering agents to improve prediction quality in the grouplens research collaborative filtering system," in Proc. the 1998 
ACM Conference on Computer Supported Cooperative Work, ACM, November 1998, pp. 345-354.

[16] S. Shishehchi, S. Y. Banihashem, N. A. M Zin, and S. A. M. Noah, "Learning content recommendation for visual basic. net programming language based on ontology," Journal of Computer Science, vol. 7, no. 2, pp. 188-196, 2011.

[17] G. Adomavicius and A. Tuzhilin, "Toward the next generation of recommender systems: A survey of the state-of-the-art and possible extensions", IEEE Transaction on Knowledge and Data Engineering, vol. 17, no. 6, pp. 734-794, 2005.

[18] A. S. Ghabayen and S. A. M. Noah, "Using tags for measuring the semantic similarity of users to enhance collaborative filtering recommender systems," International Journal on Advanced Science, Engineering and Information Technology, vol. 7, no. 6, pp. 2063-2070, 2017.

[19] M. F. Alhamid, M. Rawashdeh, H. Al Osman, M. S. Hossain, and A. El Saddik, "Towards context-sensitive collaborative media recommender system," Multimedia Tools and Applications, vol. 74, no. 24, pp. 11399-11428, 2015.

[20] A. Poon, "Tourism, technology and competitive strategies," CAB Intl., Wallingford, U.K, vol. 32, no. 3, p. 78, 1993

[21] S. Stabb, H. Werther, F. Ricci et al., "Intelligent systems for tourism," IEEE Intelligent Systems, vol. 17, no. 6, pp. 53-66, 2002.

[22] J. Xia, "E-commerce product recommendation method based on collaborative filtering technology," in Proc. International Conference on Smart Grid and Electrical Automation (ICSGEA), August 2006, pp. 90-93.

[23] B. Liu, "Many facets of sentiment analysis," A Practical Guide to Sentiment Analysis, Springer, Cham, pp. 11-39, 2017.

[24] A. N. Jebaseeli and E. Kirubakaran, "A survey on sentiment analysis of (product) reviews," International Journal of Computer Applications, vol. 47, no. 11, 2012.

[25] M. Darwich, S. A. M. Noah , and N. Omar, "Automatically generating a sentiment lexicon for the Malay language," Asia-Pacific Journal of Information Technology and Multimedia, vol. 5, no. 1, pp. 49-59, 2016.

[26] M. Darwich, S. A. M. Noah, and N. Omar, "Minimally-supervised sentiment lexicon induction model: A case study of malay sentiment analysis," in Proc.11th International Workshop on Multi-disciplinary Trends in Artificial Intelligence, 2017, vol. 10607, pp. 225-237.

[27] T. Al-Moslmi, N. Omar, S. Abdullah, and M. Albared, "Approaches to cross-domain sentiment analysis: A systematic literature review," IEEE Access, vol. 5, pp. 16173-16192, 2017.

[28] M. Hu and B. Liu, "Mining and summarizing customer reviews," in Proc. the Tenth ACM SIGKDD International Conference on Knowledge Discovery and Data Mining, August 2004, pp. 168-177.

[29] R. Guimarães, D. Z. Rodríguez, R. L. Rosa, and G. Bressan, "Recommendation system using sentiment analysis considering the polarity of the adverb," in Proc. 2016 IEEE International Symposium on Consumer Electronics (ISCE), Sao Paulo, 2016, pp. 71-72.
[30] K. Goldberg, T. Roeder, D. Gupta, and C. Perkins," Eigentaste: A constant time collaborative filtering algorithm," Information Retrieval, vol. 4, no. 2, pp. 133-151, 2001.

[31] T. Miranda, M. Claypool et al., "Combining content-based and collaborative filters in an online newspaper," in Proc. ACM SIGIR Workshop on Recommender Systems, 1999.

[32] P. Cotter and B. Smyth, "Ptv: Intelligent personalised tv guides," AAAI/IAAI, pp. 957-964, July 2005.

[33] A. Bessa, A. H. Laender, A. Veloso, and N. Ziviani, "Alleviating the sparsity problem in recommender systems by exploring underlying user communities," 2012.

[34] M. Grčar, D. Mladenič, B. Fortuna, and M. Grobelnik, "Data sparsity issues in the collaborative filtering framework," in Proc. International Workshop on Knowledge Discovery on the Web, Springer, Berlin, Heidelberg, August 2005, pp. 58-76.

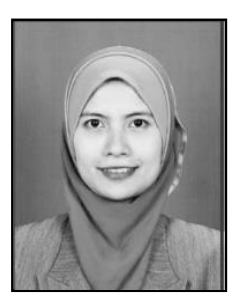

Nurul Aida Osman has received her master's degree in information technology from Universiti Kebangsaan Malaysia in 2012. Her current research work is focused on sentiment analysis and recommender systems. Her other research interest is also in the area of knowledge engineering and ontology. She is currently completing her $\mathrm{PhD}$ in computer science from Universiti Kebangsaan Malaysia.

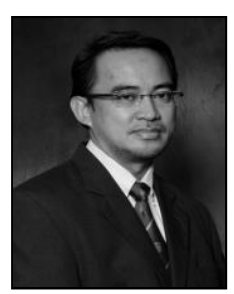

Shahrul Azman Mohd Noah received his MSc and $\mathrm{PhD}$ degrees in information studies from Sheffield University. $\mathrm{He}$ is a professor in the Faculty of Information Science and Technology, Universiti Kebangsaan Malaysia and currently heads the Knowledge Technology research group. His current research work is focused on semantic computing with special emphasis on information retrieval, ontology and recommender systems.

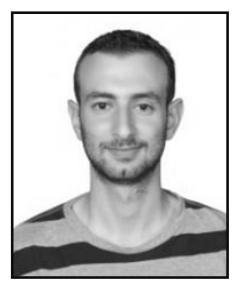

Mohammad Darwich has received his master's degree in information technology from Universiti Kebangsaan Malaysia in 2014. His research interests comprise sentiment analysis and emotion analysis in particular, and natural language processing and text mining in general. He is currently completing his $\mathrm{PhD}$ in computer science at Universiti Kebangsaan Malaysia 